\title{
GSR-TDMA: A Geometric Spatial Reuse-Time Division Multiple Access MAC Protocol for Multihop Underwater Acoustic Sensor Networks
}

\author{
Changho Yun and Yong-Kon Lim \\ Ocean System Research Division, Korea Research Institute of Ships and Ocean Engineering (KRISO), \\ No. 32, Yuseong-daero, 1312beon-gil, Yuseong-gu, Daejeon 305-343, Republic of Korea \\ Correspondence should be addressed to Changho Yun; sgn0178@kriso.re.kr
}

Received 5 October 2015; Revised 6 December 2015; Accepted 10 December 2015

Academic Editor: Davide Brunelli

Copyright ( 2016 C. Yun and Y.-K. Lim. This is an open access article distributed under the Creative Commons Attribution License, which permits unrestricted use, distribution, and reproduction in any medium, provided the original work is properly cited.

The nonnegligible propagation delay of acoustic signals causes spatiotemporal uncertainty that occasionally enables simultaneous, collision-free packet transmission among underwater nodes (UNs). These transmissions can be handled by efficiently managing the channel access of the UNs in the data-link layer. To this end, Geometric Spatial Reuse-TDMA (GSR-TDMA), a new TDMA-based MAC protocol, is designed for use in centralized, multihop underwater acoustic sensor networks (UASNs), and in this case all UNs are periodically scheduled after determining a geometric map according to the information on their location. The scheduling strategy increases the number of UNs that send packets coincidentally via two subscheduling configurations (i.e., interhop and intrahop scheduling). Extensive simulations are used to investigate the reception success rate (RSR) and the multihop delay (MHD) of GSR-TDMA, and the results are compared to those of previous approaches, including C-MAC and HSR-TDMA. GSR-TDMA outperforms C-MAC; the RSR of GSR-TDMA is 15\% higher than that of C-MAC, and the MHD of GSR-TDMA is 30\% lower than that of C-MAC at the most. In addition, GSR-TDMA provides even better performance improvements over HSR-TDMA; the RSR of GSR-TDMA is $50 \%$ higher than that of HSR-TDMA, and the MHD of GSR-TDMA is an order of $10^{2}$ lower than that of HSR-TDMA at the most.

\section{Introduction}

A number of national or multinational projects have focused on developing underwater technologies for applications related to underwater exploration, observation, investigation, surveillance, construction, mining, or excavation $[1,2]$. An underwater acoustic sensor network (UASN) is an enabling technology that can be used for these applications [1], and it is commonly used for underwater nodes (UNs) to purposefully sense and gather data and transfer that data to a land station via a surface station. Without loss of generality, a UASN is more challengeable due to the severity of the underwater environment than a radio frequency- (RF-) based terrestrial wireless sensor network (TWSN). The harsh underwater environment, which affects communications due to the extended multipath, refractive properties of medium, severe fading, and large Doppler shifts, causes high bit-error-rate and low throughput [3].
In addition, the use of acoustic signals results in a nonnegligible propagation delay that is $10^{5}$ times higher than that of RF or optical signals $[4,5]$. This long propagation delay can deteriorate the performance of the UASN (e.g., poor channel utilization, considerable latency, and frequent network failure). On the other hand, the propagation delay of the acoustic signals also leads to spatiotemporal uncertainty, which is a noticeable physical phenomenon. Although multiple acoustic signals destined to the same receiver are simultaneously sent by transmitters at different locations, collisions are not always expected due to the difference in the propagation delay of each signal [6]. This phenomenon is opposite to that of a RF-based TWSN, where coincident transmissions inevitably cause collisions, regardless of their location. Hence, an underwater medium can be spatially reused, providing that the transmission time of each UN is efficiently scheduled by considering the spatiotemporal uncertainty, and this can be achieved with the use of an 
adequate medium access control (MAC) protocol in the datalink layer.

Underwater MAC protocols are broadly divided into contention-free and contention-based MAC protocols. In the case of multihop UASNs with periodic uplink and downlink traffic, contention-based MAC protocols implemented through random access (i.e., ALOHA) or reservation via control packets (i.e., carrier sense multiple accesscollision avoidance) can be ineffective due to the increase in collisions and redundant control signaling overhead [710]. Contention-free MAC protocols include time division multiple access (TDMA), frequency division multiple access (FDMA), and code division multiple access (CDMA). FDMA is known to be the least favorable choice for a UASN because of limited bandwidth in the acoustic signals [11]. CDMA can improve throughput by using different spreading codes, but the system used to implement CDMA can be complex, and CDMA also results in a near-far problem such that a dynamic power control is additionally required $[4,12,13]$. In contrast, TDMA can be easily implemented by using simple hardware, and it is free of a near-far problem [14]. Thus, in this paper we narrow our scope to contention-free TDMA-based MAC protocols.

In the literature, several TDMA-based MAC protocols that take advantage of the spatiotemporal uncertainty have been proposed [15-18]. These MAC protocols are categorized into two types. One type finds an optimal solution by defining conflict-free constraints and formulating time scheduling. In [15], for example, the UNs execute multiple coincident transmissions with collision avoidance in a distributed manner by designing STUMP. A UN uses a Bellman-Ford distributed algorithm to find an optimal link according to its local neighbors' link schedules and propagation delay estimates. Simulation results show that STUMP outperforms TDMA, ALOHA, and T-Lohi in terms of the throughput. In [16], a TDMA-based MAC scheduling method was proposed for use in a single-hop fixed UASN. A BS formulates the MAC scheduling with four conflict-free constraints and determines the start time of all UNs to minimize interframe delays and maximize throughput.

The other type provides heuristic and conflict-free time scheduling. In [17], Cellular-MAC (C-MAC) considers a multihop UASN which consists of multiple hexagonal cells like a beehive. In C-MAC, one cycle consists of 7 time slots. At the initialization stage, a sink node determines the collisionfree time slot for all nodes by considering their location in a beehive-like network. The simulation results show that the throughput for C-MAC is higher than that for SlottedFAMA. In [18], HSR-TDMA, which is a spatial reuse TDMA with CDMA, was proposed for use in a distributed multihop UASN. An HSR-TDMA time frame consists of multiple time slots whose number is the same as the number of network nodes. For example, node $i$ becomes a slot node (i.e., a transmitting node) at time slot $i$, and node 1 becomes a slot node at time slot 1 . At time slot $j$, node $i$ calculates its hop counts from slot node $j$ and determines whether it transmits or receives at time slot $j$. This suboptimal approach enables nodes to operate in a collision-free manner with simultaneous transmissions, and simulation results show that HSR-TDMA outperforms TDMA.

In this paper, Geometric Spatial Reuse-TDMA (GSRTDMA), which is a new heuristic TDMA-based MAC protocol, is designed for use in a centralized multihop UASN. In GSR-TDMA, the medium is spatially reused by predicting the propagation delays of all UNs according to the information on their location and then transmitting packets simultaneously without collisions. To do this, the UN regularly sends its location information and that of others, which is relayed by its previous hop and finally arrived to the UN, to a base station (BS) on the surface. Then, the BS, as a central controller, can build a geometric map that contains the location information of all UNs and can thus show the overall network topology. The BS uses the geometric map to determine the transmission time of all UNs, and the scheduling of GSR-TDMA consists of two subscheduling configurations (i.e., interhop and intrahop scheduling) which increase the number of UNs sending data coincidentally. The interhop scheduling enables multiple simultaneous transmissions with collision avoidance between the UNs in different hops, and the intrahop scheduling does so between the UNs at different locations in the same hop.

The virtue of using GSR-TDMA is that collision-free transmission scheduling for all UNs can be determined simply by using regularly updated location information without requiring any complicated algorithms. In other words, it is not necessary for GSR-TDMA to determine the optimal scheduling by considering several constraints. In addition, a change in the topology can be adaptively managed by regularly updating the geometric map. Thus, GSR-TDMA can improve the efficiency of channel-reuse and can thus outperform previous suboptimal methods, including C-MAC and HSR-TDMA.

The rest of this paper is structured as follows. In Section 2, GSR-TDMA is explained in detail. In Section 3, the performance of GSR-TDMA is investigated through a simulation, and the results are compared to those of previous approaches. In Section 4, this paper is concluded.

\section{GSR-TDMA}

As illustrated in Figure 1, GSR-TDMA includes several steps. The BS primarily sets or updates the geometric map. Then, the BS uses the geometric map to determine the interand intrahop scheduling. Finally, the BS sets all timing parameters, including the transmission time offsets of all UNs, the transmission cycle, and the cycle start time.

2.1. Setting the Geometric Map. The geometric map is primarily set when the network is initialized, with the condition that the BS knows the information on the initial location of all UNs, and the BS periodically updates the geometric map after receiving all the location information gathered from the UNs that had been transmitted together with the data. The manner in which a geometric map is set is given as follows:

(i) The BS locates the UNs in a $2 \mathrm{D}$ plane according to their location information. The BS locates itself in the center, and the UNs are spread around the BS in a $2 \mathrm{D}$ plane. 


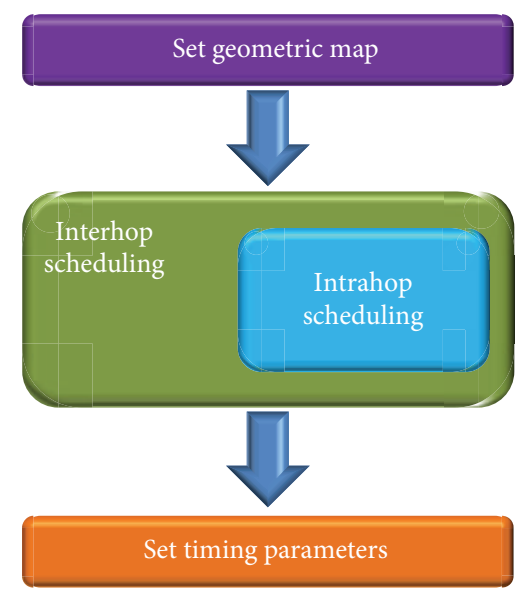

FIGURE 1: The concept of GSR-TDMA.

(ii) The $2 \mathrm{D}$ plane is divided into several zones with the BS as the center. The zones include one circle and multiple rings, as shown in Figure 2. Throughout this paper, let us rename a zone as a hop. Both the radius of the circle and the thickness of a ring are given as the maximum communication range $(R)$. We attach a hop number, $h$, to each hop according to the distance from the BS $\left(L_{\mathrm{BS}}\right)$ which is the multiple of $R$. Hop $0(h=0)$ is just the BS, and Hop $1(h=1)$ is the circle including the UNs for which $L_{\mathrm{BS}}$ is less than $R$. Likewise, Hop $2(h=2)$ is the ring that includes the UNs for which $L_{\mathrm{BS}}$ is less than $2 R$ but more than $R$.

(iii) The BS maintains the hop number for all UNs in order to schedule their transmission time offsets.

2.2. Interhop Scheduling. If the hop number $(h)$ difference between any two UNs is more than three, they are collisionfree in spite of performing simultaneous transmissions. This idea implies that all hops of the geometric map are categorized into three interhop groups $\left(\mathrm{IHG}_{n}, 0 \leq n \leq 2\right)$. As a result, collision-free transmissions are guaranteed among the several UNs that are located at different hops belonging to the same intergroup. An intergroup can be defined as a set of collision-free hops. Let us define $m$ as the number of hops. Let us also define $x$ and $y$ as $x=\lfloor m / 3\rfloor$ and $y=\bmod (m, 3)$. Using $x$ and $y, \mathrm{IHG}_{n}$ can be expressed as

$$
\begin{aligned}
& \mathrm{IHG}_{n} \\
& = \begin{cases}{[0,3, \ldots, 3 x-3,3 x],} & \text { for } n=0, \\
{[n, n+3, \ldots, 3 x-3+n, 3 x+n],} & \text { for } 1 \leq n \leq 2, n \leq y, \\
{[n, n+3, \ldots, 3 x-3+n],} & \text { for } 1 \leq n \leq 2, n>y .\end{cases}
\end{aligned}
$$

There are two possible scheduling scenarios: one is where there is an uplink order $\left(\mathrm{IHG}_{2} \rightarrow \mathrm{IHG}_{1} \rightarrow \mathrm{IHG}_{0}\right)$, and the other is where there is a downlink order $\left(\mathrm{IHG}_{0} \rightarrow \mathrm{IHG}_{1} \rightarrow\right.$ $\mathrm{IHG}_{2}$ ). The direction of the interhop scheduling depends on a target application. For an example, if the BS just periodically broadcasts a control packet to all UNs and the UNs mainly send the sensed data to the BS, the uplink interhop scheduling

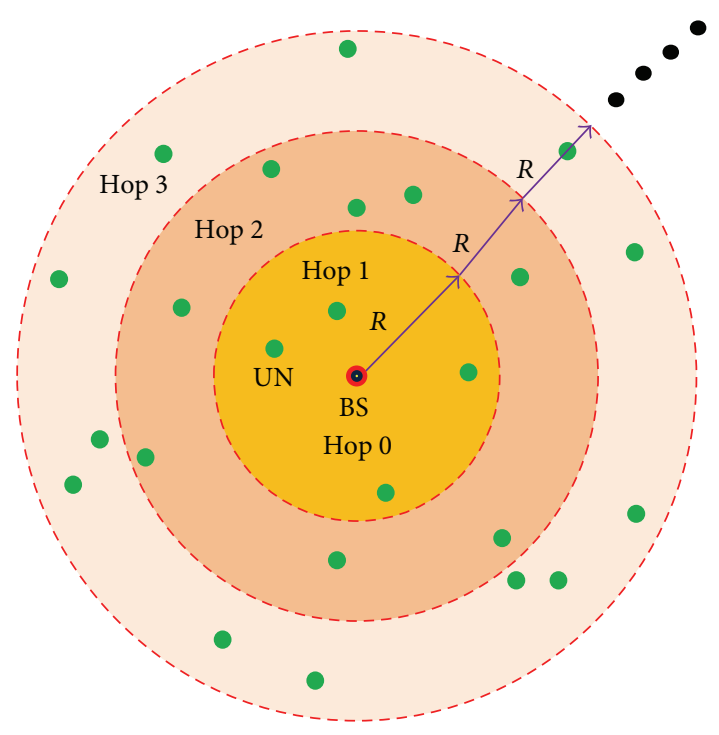

FIGURE 2: An example of a geometric map.

can be efficient. On the other hand, we can interchangeably decide the direction of the interhop scheduling according to the instantaneous frequency and the volume of the uplink and downlink traffic.

2.3. Intrahop Scheduling. The intrahop scheduling involves all UNs that belong to the same hop, while the interhop scheduling involves all hops belonging to the same interhop group. The intrahop scheduling is applied to hops where collision-free and coincident transmissions of the UNs are available $(h \geq 2)$. In the intrahop scheduling, a hop is divided into several subhops, as illustrated in Figure 3. The way to divide the hop into multiple subhops is given as follows:

(i) Hop $h$ is divided into $2^{h}$ subhops (Subhop $1, \ldots$, Subhop $2^{h}$ ).

(ii) A UN with the shortest $L_{\mathrm{BS}}$ is selected as a reference $\mathrm{UN}$ in Hop $h$.

(iii) A line between the reference UN and the BS (a reference line) is drawn vertical to the reference line. This results in four arcs per hop.

(iv) Each arc is divided into equal $2^{h-2}$ arcs around the BS in order to obtain $2^{h}$ subhops.

(v) We define the subhop to be where a reference UN is located as a reference subhop.

(vi) As Hop 2 in Figure 3 exemplifies, half $\left(2^{h-1}\right)$ of all subhops can be collision-free in a hop because nonneighboring subhops, which are one subhop apart, are collision-free. Let us then define $k$ as the subhop number. Starting with the reference subhop, all collision-free subhops are numbered as $1,2, \ldots, 2^{h-1}$. These subhops belong to an upper subhop group $\left(\mathrm{SHG}_{U}\right)$. The other half are also collision-free and are numbered as $2^{h-1}+1,2^{h-1}+2, \ldots, 2^{h}$. These subhops belong to a lower subhop group $\left(\mathrm{SHG}_{L}\right)$. 


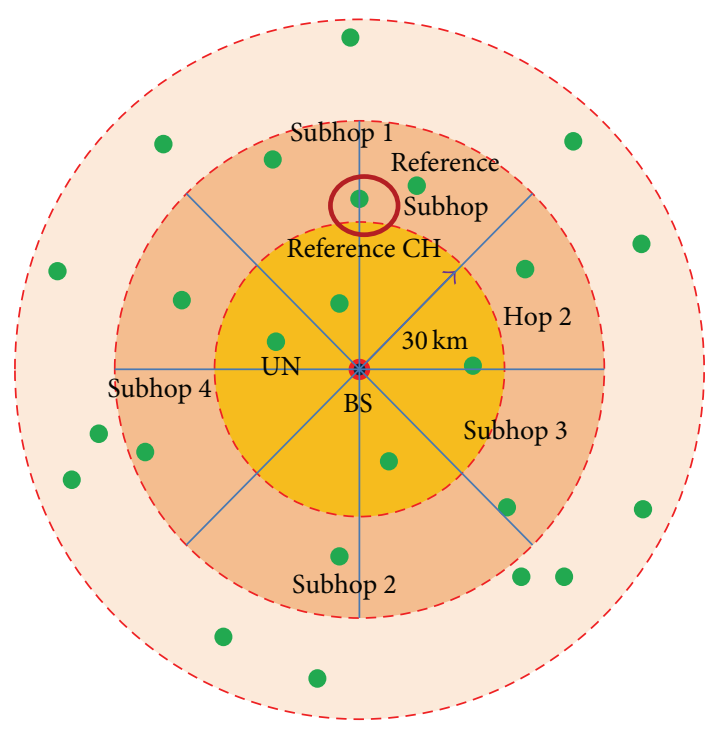

FIgURE 3: An example of dividing a hop into multiple subhops.

Based on the concept of subhops, the intrahop scheduling is given as follows:

(i) In Hop 1 where collision-free and coincident transmissions for the UNs are unavailable, the transmission order of the UNs is determined in the $L_{\mathrm{BS}}$ descending order.

(ii) In Hop $h(h \geq 2), \mathrm{SHG}_{U}$ sends first and $\mathrm{SHG}_{L}$ does last. In a subhop, the transmission order of UNs is also given as $L_{\mathrm{BS}}$ descending order.

Figure 4 illustrates an example with the intrahop scheduling for hops belonging to $\mathrm{IHG}_{0}$ in the case of the downlink ordered interhop scheduling.

2.4. Setting Timing Parameters. Here we formulate two timing parameters by considering the inter- and intrascheduling, including the transmission time offsets for all UNs and the transmission cycle. The concept for the timing parameters is exemplified in Figure 5. The definitions of all parameters in order to define the timing parameters are given at the end of the paper. Let us consider a UN $j$ that belongs to Subhop $k$ in Hop $h$. The UN $j$ should wait during its waiting time, $W(h, k, j)$, and should then send packets during its service time, $S(h, k, j)$. Since the UN $j$ can send packets right after several UNs that belong to the same subhop as the UN $j$ but send packets earlier than the UN $j$, the finished transmission, $W(h, k, j)$, is expressed as

$$
\begin{aligned}
& W(h, k, j) \\
& = \begin{cases}(j-1) \times\left(\mathrm{LEN}_{h} \times \mathrm{TD}+T_{G}\right)+\sum_{p=1}^{j-1} \mathrm{PD}_{h, k, p}, & \text { for } j>1 \\
0, & \text { for } j=1 .\end{cases}
\end{aligned}
$$

$S(h, k, j)$ is also represented as

$$
S(h, k, j)=\mathrm{LEN}_{h} \times \mathrm{TD}+T_{G}+\mathrm{PD}_{h, k, j} .
$$

Using (2) and (3), the time when all UNs belonging to Subhop $k$ in Hop $h$ send packets can be expressed as $W\left(h, k, N_{h, k}\right)+$ $S\left(h, k, N_{h, k}\right)$, as illustrated in Figure 5. Since there are $2^{h-1}$ subhops in $\mathrm{SHG}_{U}$ for Hop $h$, the maximum for $W\left(h, k, N_{h, k}\right)+$ $S\left(h, k, N_{h, k}\right)$ in $\mathrm{SHG}_{U}, f_{U}(h)$, is expressed as

$$
\begin{aligned}
f_{U}(h)=\max \left\{W\left(h, k, N_{h, k}\right)+S\left(h, k, N_{h, k}\right)\right\} & \\
k & \in \mathrm{SHG}_{U} .
\end{aligned}
$$

Similarly, the maximum for $W\left(h, k, N_{h, k}\right)+S\left(h, k, N_{h, k}\right)$ in $\mathrm{SHG}_{L}, f_{L}(h)$, is expressed as

$$
\begin{aligned}
f_{L}(h)=\max \left\{W\left(h, k, N_{h, k}\right)+S\left(h, k, N_{h, k}\right)\right\} & \\
k & \in \mathrm{SHG}_{L} .
\end{aligned}
$$

If we define the time for all UNs belonging to Hop $h$ in $\mathrm{IHG}_{n}$ as $f_{U}(h)+f_{L}(h)$, the maximum of $f_{U}(h)+f_{L}(h)$ in $\mathrm{IHG}_{n}$, $F(n)$, is represented as

$$
F(n)=\max \left\{f_{U}(h)+f_{L}(h)\right\}, \quad h \in \mathrm{IHG}_{n} .
$$

Using (2)-(6), the transmission time offset of the UN $j$ belonging to Subhop $k$ in Hop $h$ in $\mathrm{IHG}_{n}, T(n, h, k, j)$, can be finally obtained as

$$
T(n, h, k, j)= \begin{cases}0, & \text { for } n=h=0, \\ \left\lfloor\frac{k-1}{2^{h-1}}\right\rfloor \times f_{U}(h)+W(h, k, j), & \text { for } n=0, h \in \mathrm{ING}_{0}, h \geq 3, \\ F(0)+W(1,1, j), & \text { for } n=h=1, \\ \sum_{b=0}^{n-1} F(n)+\left\lfloor\frac{k-1}{2^{h-1}}\right\rfloor \times f_{U}(h)+W(h, k, j), & \text { for } 1 \leq n \leq 2, h \in \mathrm{ING}_{n}, h \neq 1 .\end{cases}
$$

The transmission cycle is the same as the time when one interhop scheduling is executed. Thus, the transmission cycle, $T_{\text {cycle }}$, is expressed as 


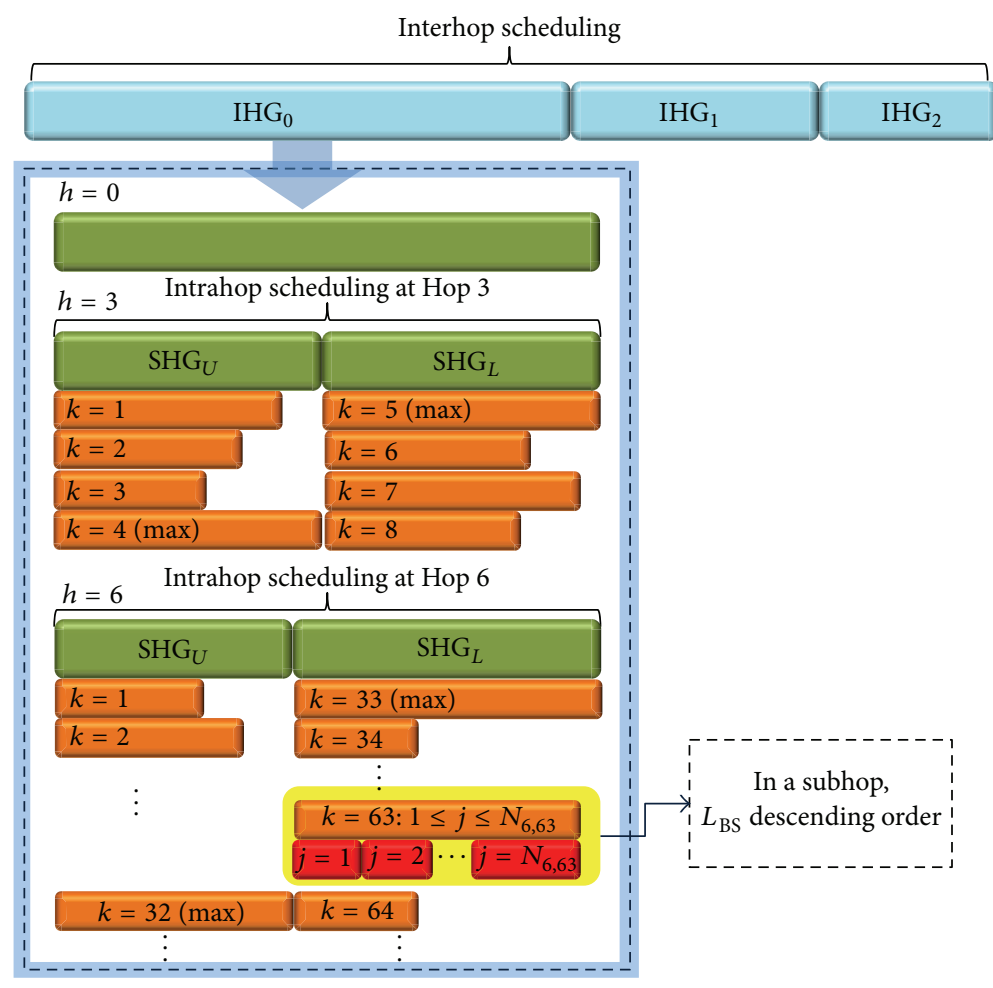

FIGURE 4: The concept of inter- and intrahop scheduling.

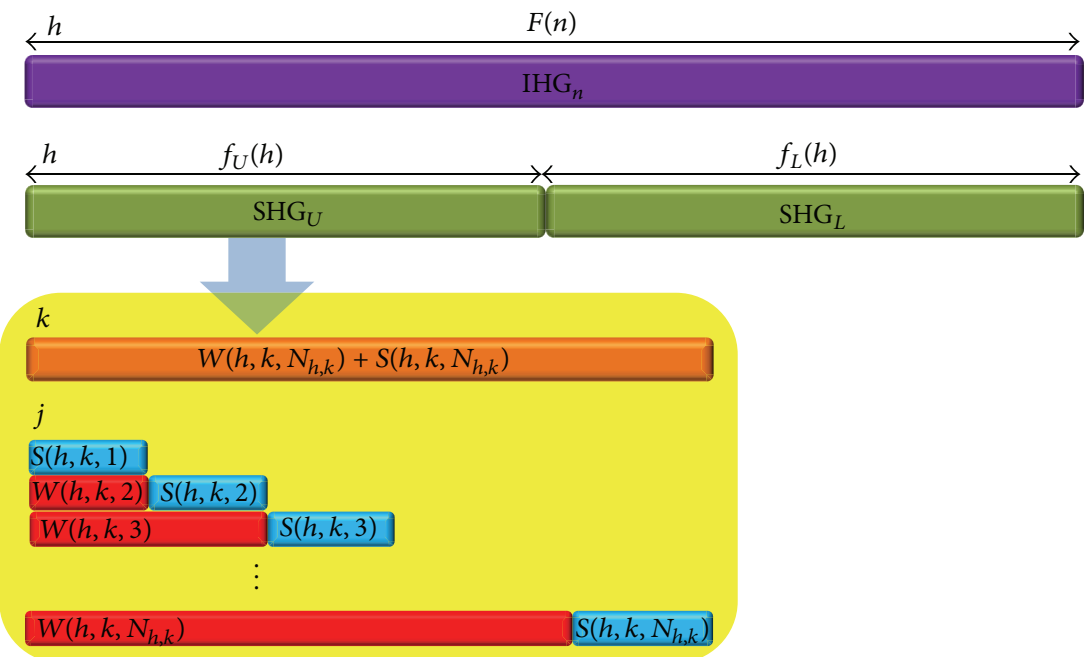

FIGURE 5: The concept of determining the timing parameters.

The BS informs all UNs of all timing parameters, including $T(n, h, k, j), T_{\text {cycle }}$, and $T_{0}$, which is the initial time point for the transmission of the packet. Using these timing parameters, all UNs can access the channel for each cycle. Namely, the UN can transmit packets after waiting for $T(n, h, k, j)$ starting from $(p-1) \times T_{\text {cycle }}$ at cycle $p$.

\section{Simulation Results}

In this section, the performance of GSR-TDMA is investigated through the use of simulations. The simulator is event-driven, and multiple events occur at the same time at one discrete simulation time point $t(1 \leq t \leq T)$, where $T$ is the total duration of the simulation. In addition, 100 random experiments are executed for each case in order to obtain averaged performance results. follows:

The network conditions for the simulations are given as

(i) A fixed and centralized multihop network topology is used in which a BS is located at the center, and multiple UNs are deployed, as shown in Figure 2.

(ii) The maximum propagation distance $(R)$ is $10 \mathrm{~km}$. 
(iii) $m$ is given as $3 \leq m \leq 7$ in order to see the effect of the number of hops.

(iv) Two UN distribution scenarios are considered according to UNs' distribution per hop. One is a simple scenario that the positions of UNs are uniformly distributed and the number of UNs gradually increases according to hops as $N_{h}=4 \times h$ $(h \geq 1)$. The other is a scenario that UNs are randomly distributed so that the number of UNs per hop is also randomly given.

(v) The uplink and downlink routes between the BS and a UN are determined upon initializing the network.

(vi) In practice, corrupted packets are hardly recovered once acoustic signals are distorted underwater, and the transmitted packets cannot even reach their destination in the worst case. Thus, an error in the simulation is modelled as that where all packet transmissions, which are scheduled at error-occurring simulation time points, fail. We consider the error rate $e$ to be the ratio of the amount of error-occurring simulation durations over $T$. An error is randomly given to any $\mathrm{UN}$ at any $t$, and $e$ is given as $e=$ $0: 0.2: 0.8$.

The traffic conditions are given as follows:

(i) Both uplink and downlink traffic are generated.

(ii) One packet is generated, and the period of the packet generation $(P)$ is $P=\{3,5,10,25\} . P=3$ implies that all UNs and BS generate a packet every third simulation time point, that is, when $t=\{1,4,7, \ldots, T\}$.

(iii) The number of packets served per one transmission or the size of the packet train ( $\mathrm{SN})$ is given as $\mathrm{SN}=$ $1: 2: 15$. The length of a packet is of 1000 bits, and the data rate is given as $1000 \mathrm{bps}$.

Two performance parameters are considered, and these include the reception success rate (RSR) and the multihop delay (MHD). The RSR is the ratio of the number of packets received to that of packets transmitted. Two RSR parameters are used, one for uplink $\left(\mathrm{RSR}_{U}\right)$ and one for downlink $\left(\mathrm{RSR}_{D}\right)$. In order to define the RSR, let us define $\mathrm{RN}_{\mathrm{BS}}$ as the number of packets received at the $\mathrm{BS}$ sent by all $\mathrm{UNs}, \mathrm{TN}_{\mathrm{UN} i}$ is the number of packets transmitted from a UN $i$ to a BS, $\mathrm{TN}_{\mathrm{BS} i}$ is the number of packets transmitted from a BS to a $\mathrm{UN} i$, and $\mathrm{RN}_{\mathrm{UN} i}$ is the number of packets received at a $\mathrm{UN} i$ sent by a $\mathrm{BS}$ at a $\mathrm{UN} i$, respectively. Then, the RSR is expressed for the uplink and downlink as

$$
\mathrm{RSR}= \begin{cases}\frac{\mathrm{RN}_{\mathrm{BS}}}{\sum_{i=1}^{N} \mathrm{TN}_{\mathrm{UN} i}}, & \text { for uplink, } \\ \frac{1}{N} \sum_{i=1}^{N} \frac{\mathrm{RN}_{\mathrm{UN} i}}{\mathrm{TN}_{\mathrm{BS} i}}, & \text { for downlink. }\end{cases}
$$

The MHD represents the multihop end-to-end delay from the time the packet is generated at the source to the time the packet is received at the destination. Like the RSR, there are two MHD parameters, one for uplink $\left(\mathrm{MHD}_{U}\right)$ and one for downlink $\left(\mathrm{MHD}_{D}\right)$. Let us define $\mathrm{UD}_{i}$ as the uplink end-toend delay from a UN $i$ to a BS and $\mathrm{DD}_{i}$ as the downlink endto-end delay from a BS to a UN $i$. Then, the MHD for the uplink and downlink are expressed as

$$
\mathrm{MHD}= \begin{cases}\frac{1}{N} \sum_{i=1}^{N} \mathrm{UD}_{i}, & \text { for uplink, } \\ \frac{1}{N} \sum_{i=1}^{N} \mathrm{DD}_{i} & \text { for downlink. }\end{cases}
$$

The simulation experiments are executed under the following four cases.

Case I. The experiment is error-free, and the number of hops $(m)$ varies in order to investigate the effect of $m$ when the positions of UNs are uniformly distributed and the number of UNs gradually increases according to hops.

Case II. The experiment is error-free, and the period of packet generation $(P)$ varies in order to investigate the effect of $P$ when the positions of UNs are uniformly distributed and the number of UNs gradually increases according to hops.

Case III. The experiment is error-occurring at fixed $m$ and $P$ in order to investigate the effect of $e$ when the positions of UNs are uniformly distributed and the number of UNs gradually increases according to hops.

Case IV. The experiment is error-free, and the number of hops $(m)$ varies considering random UN distribution and density in order to investigate the effect of random node distribution.

Case I. Figure 6 illustrates the RSR performance of the three MAC protocols, according to SN and $m$, when $P$ is fixed at 10 . The results indicate that the RSR of GSR-TDMA is better than that of C-MAC, regardless of $m$ and SN, for both the uplink and downlink traffic, with a maximum RSR margin of $15 \%$ (see Figure 6(b)). GSR-TDMA also outperforms HSR-TDMA in a large percentage of the cases, and the maximum RSR margin is almost $50 \%$ (see Figure 6(a)). Although the RSR of HSR-TDMA is better than that of GSR-TDMA, in the case of $\mathrm{SN}<10$ for $m>5$, the margin is unremarkable $(\sim 5 \%)$. The results also indicate that the three MAC protocols have little difference in terms of RSR (i.e., within 10\%) as $m$ increases. This occurs because the uplink and downlink traffic become heavy as the number of UNs increases with an increase in $m$. This causes the RSR of the UNs far away from a BS to decrease, and the overall RSR performance is degraded. In terms of $\mathrm{SN}$, we can intuitively expect for the RSR to be enhanced as $\mathrm{SN}$ increases. The results of the simulation indicate that the RSR improves and is then saturated at a certain value of SN. Thus, the value of SN (i.e., the size of the packet train) can be determined according to the values of $m$ and $P$. In addition, the use of GSR-TDMA is more favorable than that of the other two MAC protocols, in the case of the downlink traffic. Figures 6(d)-6(f) illustrate how GSR-TDMA outperforms HSR-TDMA and C-MAC, regardless of SN and $m$.

Figure 7 illustrates the performance for both the uplink and downlink MHD in Case I. As $m$ increases, $\mathrm{MHD}_{U}$ and 


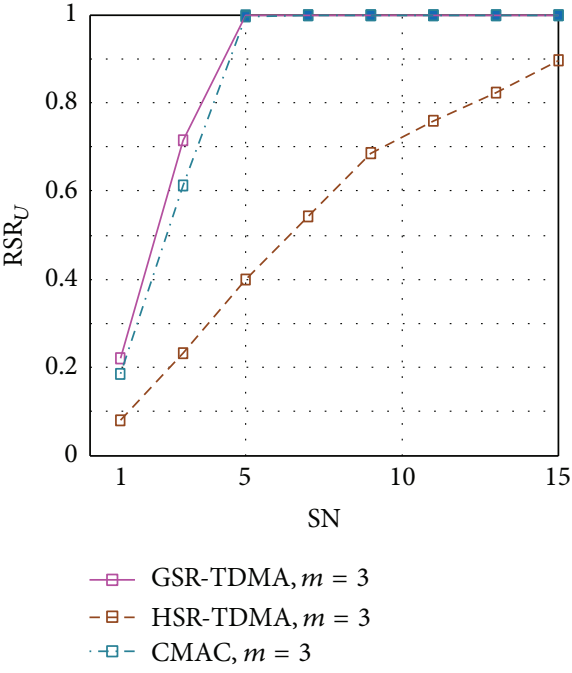

(a)
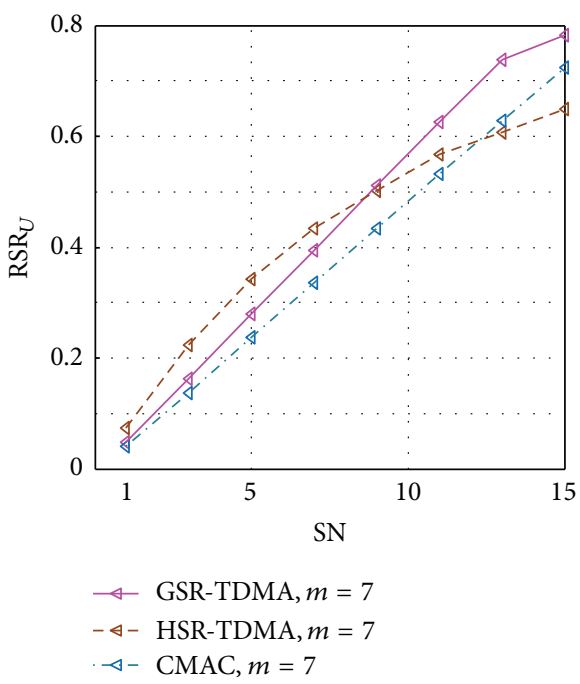

(c)

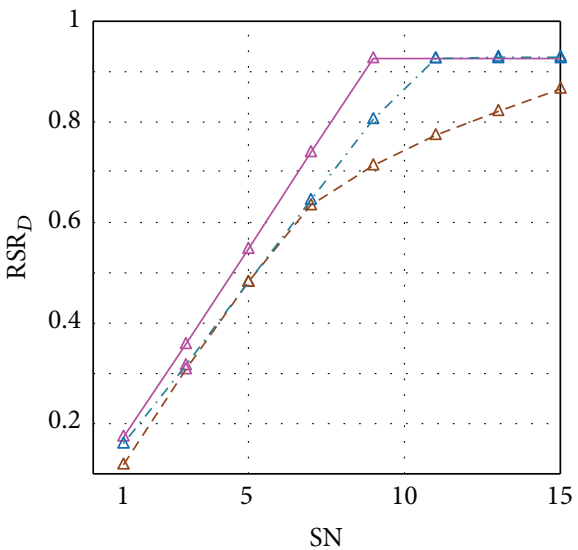

$\triangle$ GSR-TDMA, $m=5$

$-\triangle-$ HSR-TDMA, $m=5$

$\triangle-$ CMAC, $m=5$

(e)

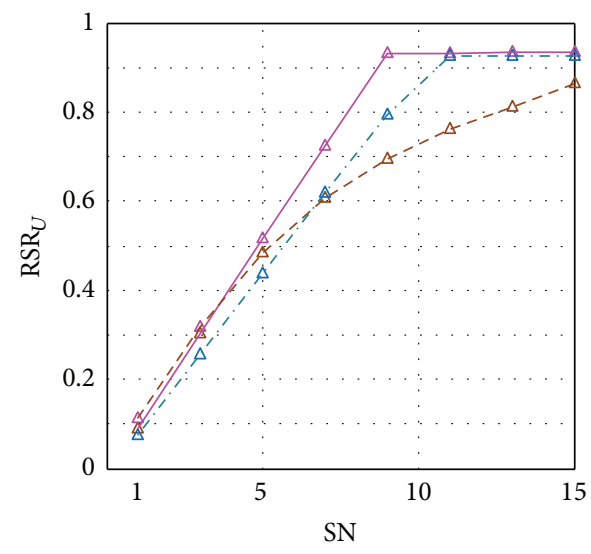

$\triangle$ GSR-TDMA, $m=5$

$-\Delta-$ HSR-TDMA, $m=5$

$-\triangle-$ CMAC, $m=5$

(b)

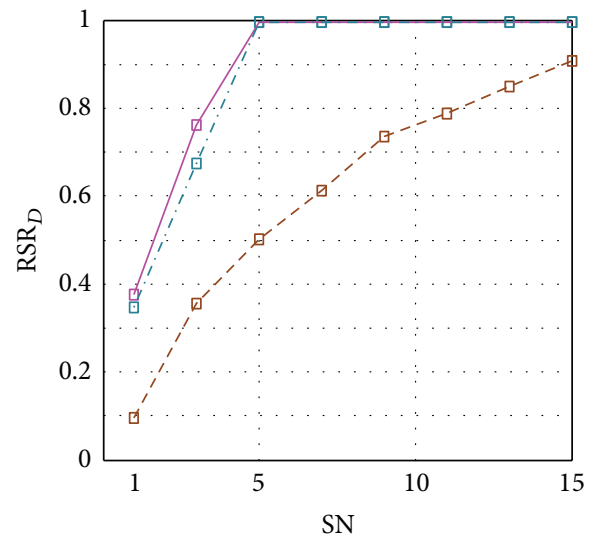

$\square$ GSR-TDMA, $m=3$

- - HSR-TDMA, $m=3$

$\square-$ CMAC, $m=3$

(d)

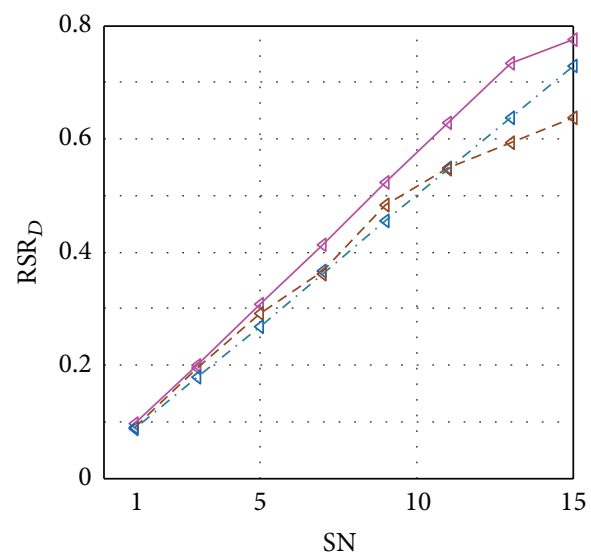

$\triangleleft$ GSR-TDMA, $m=7$

$-\triangleleft-$ HSR-TDMA, $m=7$

$\triangleleft-$ CMAC, $m=7$

(f)

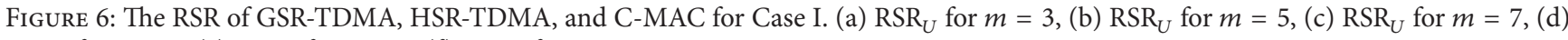
$\mathrm{RSR}_{D}$ for $m=3$, (e) $\mathrm{RSR}_{D}$ for $m=5$, (f) $\mathrm{RSR}_{D}$ for $m=7$. 

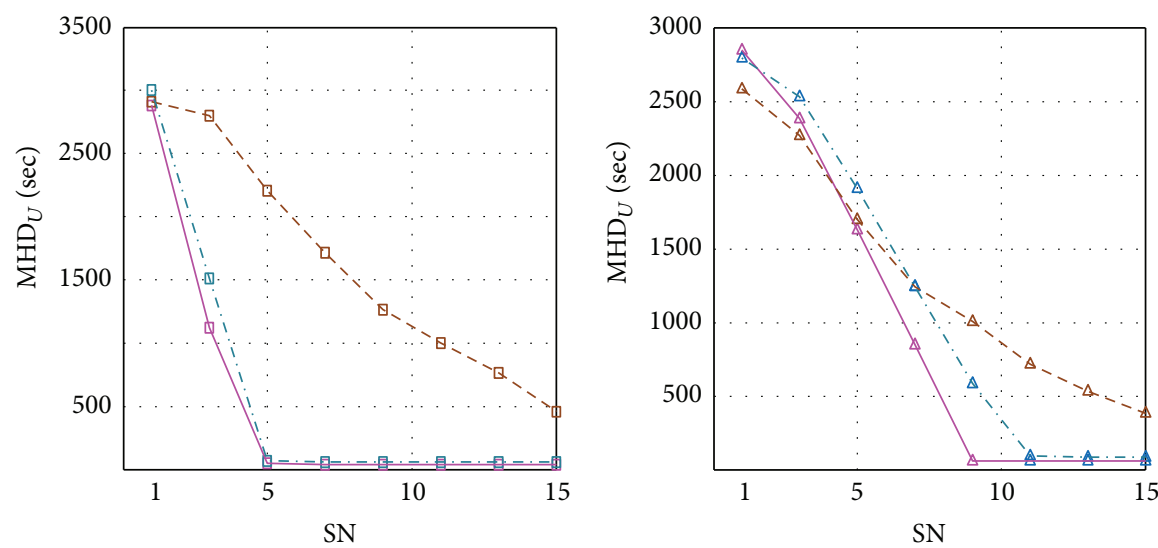

$\square$ GSR-TDMA, $m=3$
$-\boxminus-$ HSR-TDMA, $m=3$
$\cdot \square-$ CMAC, $m=3$

(a)

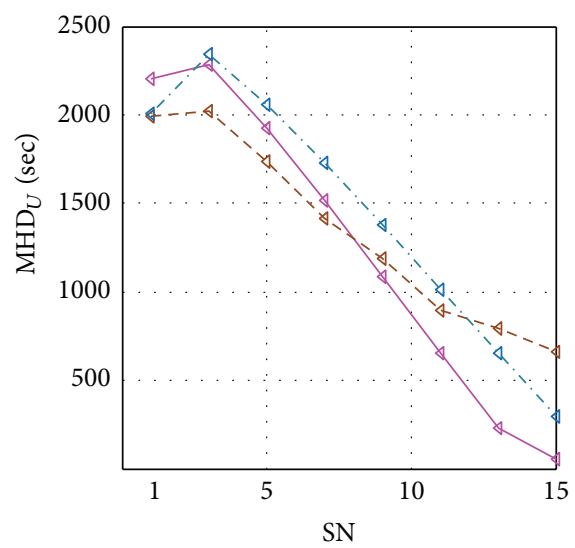

$\triangleleft$ GSR-TDMA, $m=7$

$-\triangleleft-$ HSR-TDMA, $m=7$

$\triangleleft-$ CMAC, $m=7$

(c)

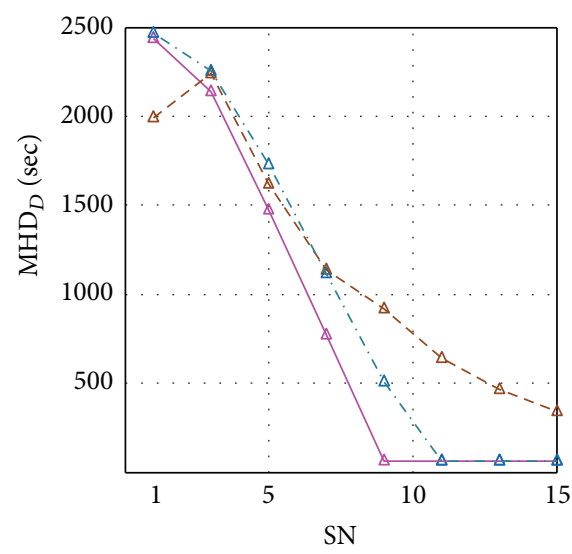

$\triangle$ GSR-TDMA, $m=5$

- $\triangle$ - HSR-TDMA, $m=5$

$\triangle-$ CMAC, $m=5$

(e)

$$
\begin{aligned}
& \triangle \text { GSR-TDMA, } m=5 \\
& -\triangle-\text { HSR-TDMA, } m=5 \\
& -\Delta-\text { CMAC, } m=5
\end{aligned}
$$

(b)

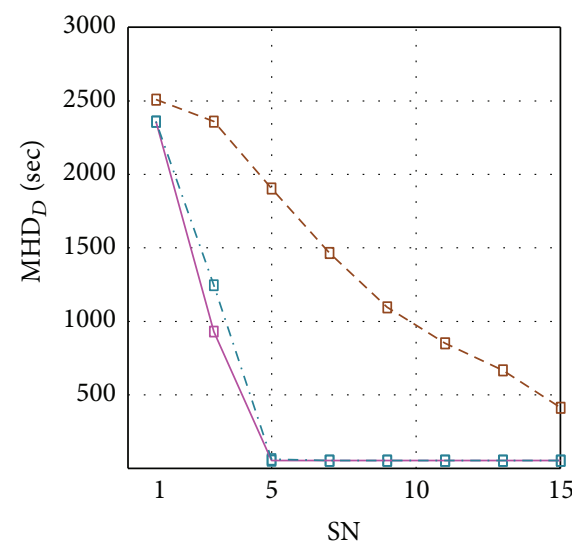

$$
\begin{aligned}
& -\square \text { GSR-TDMA, } m=3 \\
& -\boxminus-\text { HSR-TDMA, } m=3 \\
& -\square-\text { CMAC, } m=3
\end{aligned}
$$

(d)

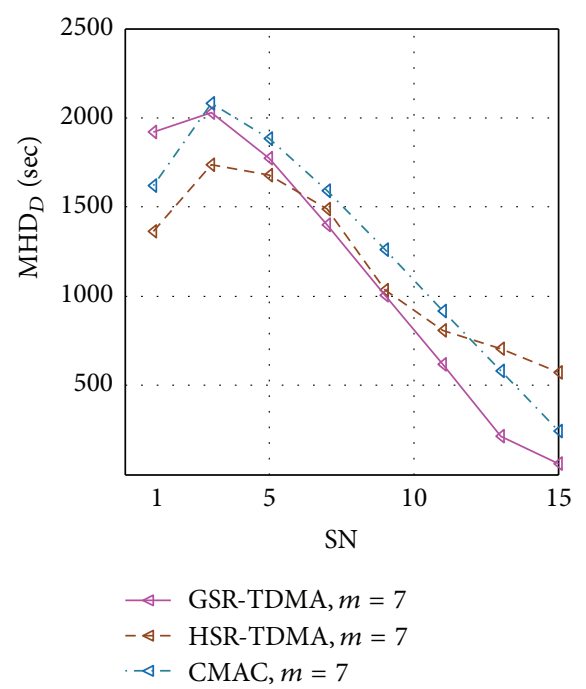

(f)

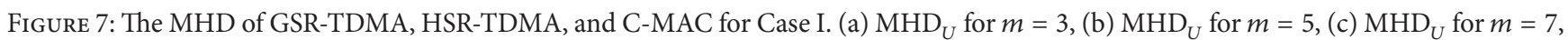
(d) $\mathrm{MHD}_{D}$ for $m=3$, (e) $\mathrm{MHD}_{D}$ for $m=5$, and (f) $\mathrm{MHD}_{D}$ for $m=7$. 

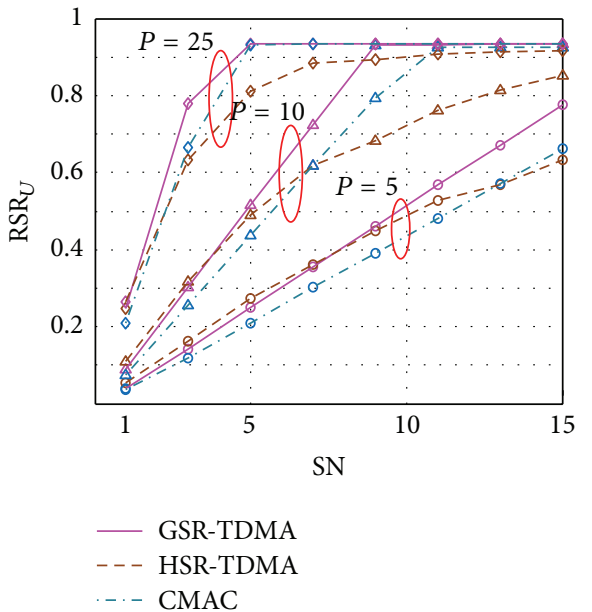

(a)

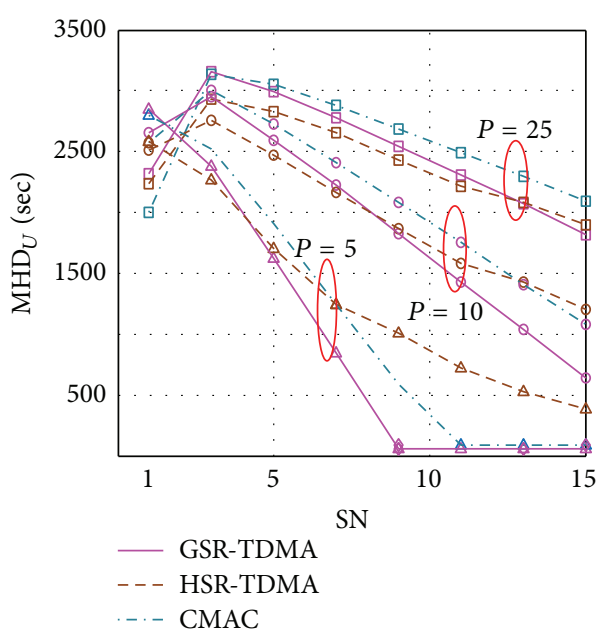

(c)

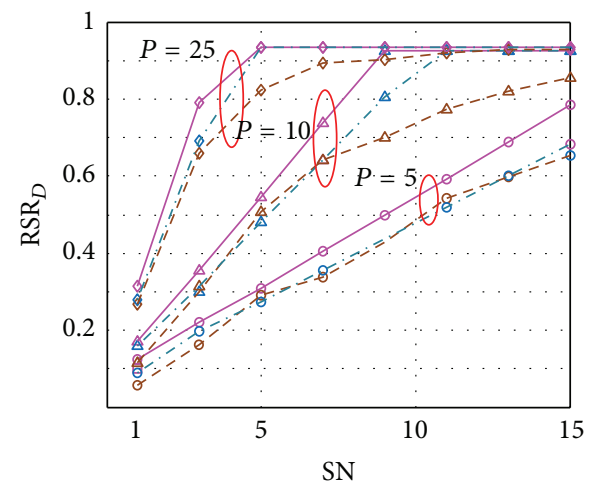

- GSR-TDMA

- - HSR-TDMA

-.. CMAC

(b)

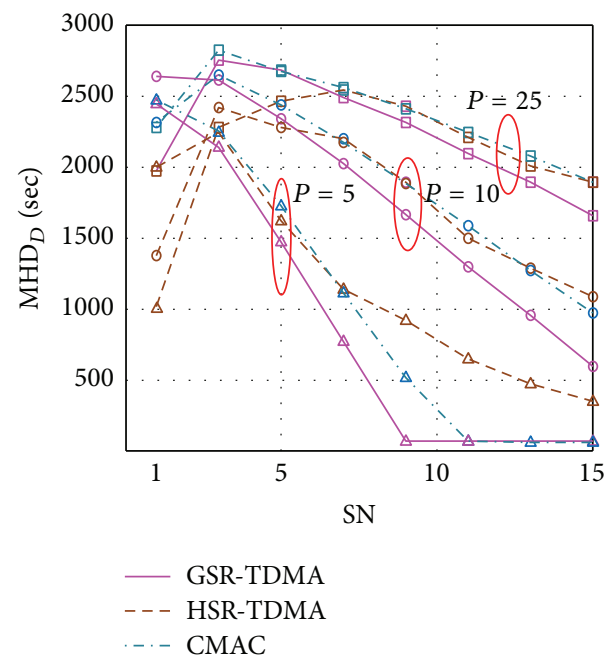

(d)

FIGURE 8: The RSR and MHD of GSR-TDMA, HSR-TDMA, and C-MAC for Case II. (a) RSR $R_{U}$, (b) RSR $R_{D}$, (c) $\mathrm{MHD}_{U}$, and (d) MHD ${ }_{D}$.

$\mathrm{MHD}_{D}$ for the three MAC protocols decrease. This result counters our intuition that the multihop end-to-end delay increases as the number of hops increases and originates from the fact that the UNs that are far away from a BS are more likely to experience transmission failures due to packet drops than those close to the BS. Thus, the multihop end-to-end delays of the UNs close to the BS are mainly a concern to the extent that the averaged $\mathrm{MHD}_{U}$ and $\mathrm{MHD}_{D}$ decrease. The MHD for GSR-TDMA is lower than that of C-MAC, regardless of $m$ and $\mathrm{SN}$, for both the uplink and downlink traffic. The MHD of GSR-TDMA is 30\% shorter than that of C-MAC at maximum. Similarly to the RSR performance, the MHD of GSR-TDMA is lower than that of HSR-TDMA in a large percentage of the cases. The MHD of GSR-TDMA is lower than that of HSR-TDMA by an order of $10^{2}$ at maximum (see Figures 7(a) and 7(d)). Although the MHD of HSRTDMA is lower than that of GSR-TDMA in the case of SN < 10 for $m>5$, the margin is less than $10 \%$.

Case II. Figure 8 illustrates the RSR and MHD for the three MAC protocols for the uplink and downlink traffic according to $\mathrm{SN}$ and $P$ when $m$ is five. The RSR improves as $P$ increases due to the increase in the queueing margin. Applying a packet train to GSR-TDMA is shown to be more efficient than applying a packet train to HSR-TDMA and C-MAC because the RSR of GSR-TDMA is better than that of HSRTDMA and C-MAC as SN increases. The RSR performance margin between GSR-TDMA and HSR-TDMA is almost 25\% at maximum (see Figures 8(a) and 8(b)).

In terms of the MHD performance, GSR-TDMA is shown to be more efficient in the use of the packet train than HSRTDMA and C-MAC because the MHD difference among the three MAC protocols is remarkable as $\mathrm{SN}$ increases. The MHD of GSR-TDMA is almost twenty times higher than that of HSR-TDMA at maximum (see Figures 8(c) and 8(d)).

Case III. Figure 9 illustrates the RSR and MHD of the three MAC protocols in the error-occurring case. For instance, an error rate of $0.2(e=0.2)$ implies that errors randomly occur for $20 \%$ of the total simulation time. A UN that is scheduled to transmit its packet train at any time point during the erroroccurring simulation duration experiences a transmission 


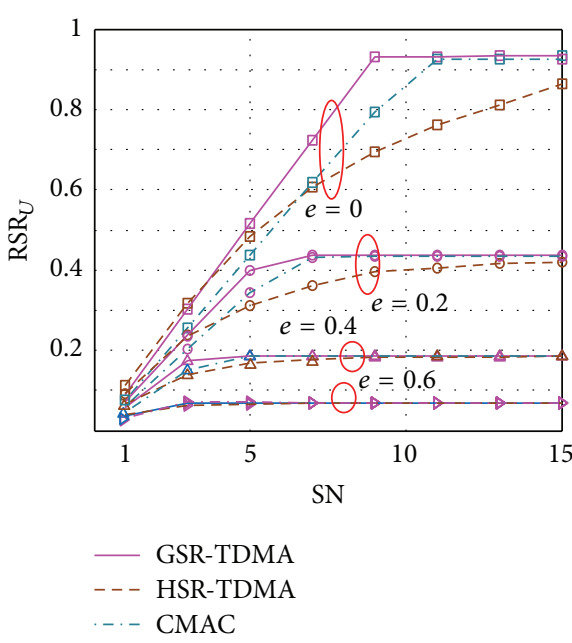

(a)

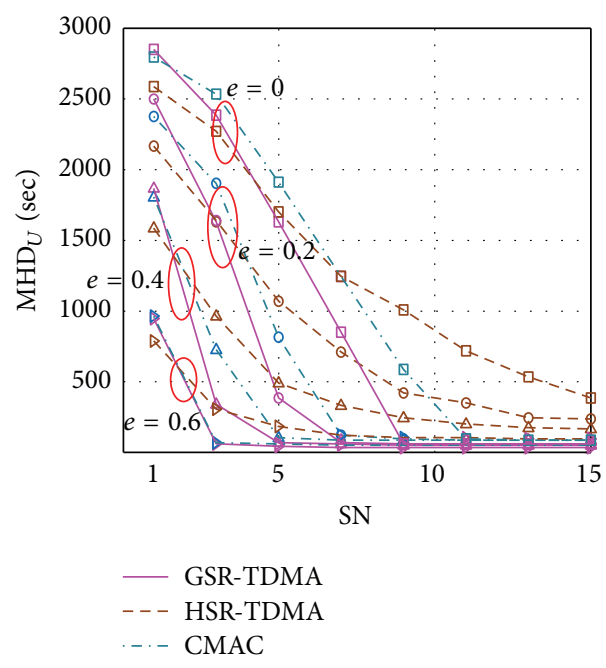

(c)

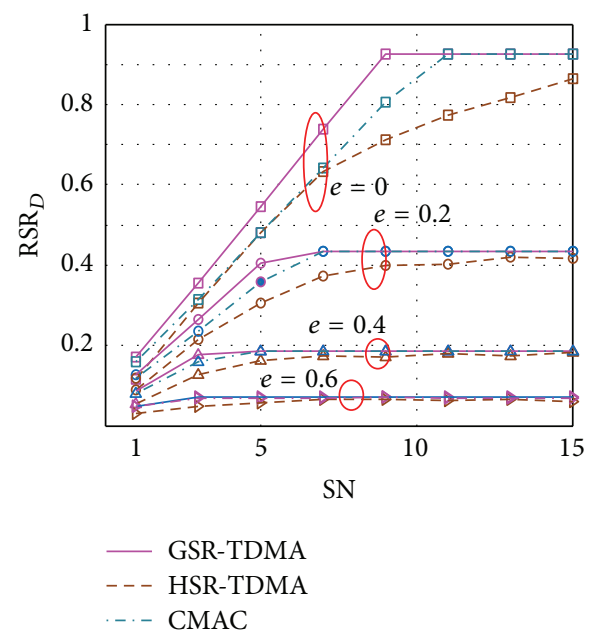

(b)

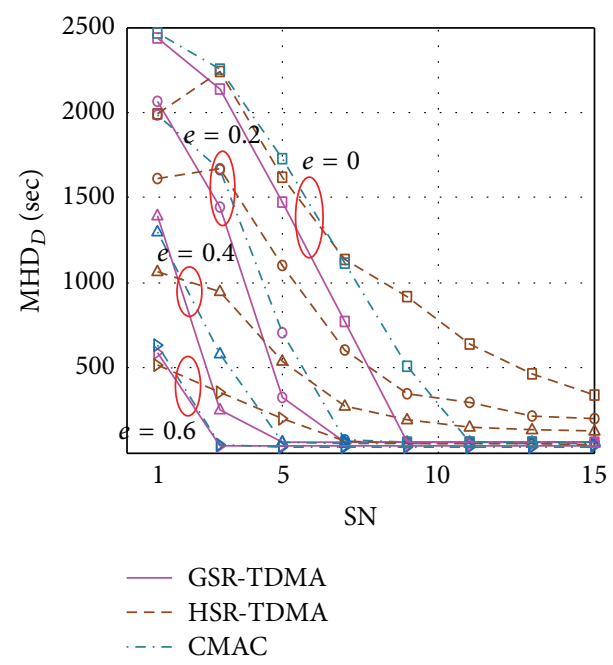

(d)

Figure 9: The RSR and MHD of GSR-TDMA, HSR-TDMA, and C-MAC for Case III. (a) RSR $\mathrm{R}_{U}$, (b) RSR $\mathrm{R}_{D}$, (c) $\mathrm{MHD}_{U}$, and (d) MHD . $^{\text {. }}$

failure. We also fix the other parameters, with $m=5$ and $P=1$, where GSR-TDMA outperforms HSR-TDMA and CMAC in terms of RSR and MHD. The results indicate that the RSR severely deteriorates as $e$ increases. There is no rebound in the performance among the three MAC protocols, and the performance superiority still holds, regardless of $e$. The performance margin becomes insignificant as $e$ increases due to the frequency of the transmission failures. These results still hold in the case of different values of $m$ and $P$. On the other hand, as $e$ increases, the UNs that are far away from the BS are prone to suffer from transmission failures by going through several hops. Thus, the multihop end-to-end delays of the UNs close to a BS are mainly a concern to the extent that the averaged $\mathrm{MHD}_{U}$ and $\mathrm{MHD}_{D}$ finally decrease, as shown in Figures 9(c) and 9(d).

Case IV. Figure 10 illustrates the RSR performance of the three MAC protocols, according to $\mathrm{SN}$ and $m$, when $P$ is fixed at 10 and UNs are randomly distributed and the number of UNs according to hops is also random. As shown in Figures 10(a), 10(b), 10(d), and 10(e), the RSR of GSR-TDMA is mostly lower than that of C-MAC with slight margin when $m$ is less than five. This is because the possibility of coincident TDMA scheduling among UNs in GSR-TDMA may decrease under UNs' random distribution and density. On the contrary, GSRTDMA can be still advantageous when the number of hops is greater than five, as shown in Figures 10(c) and 10(f), showing that the RSR of GSR-TDMA is greater than that of C-MAC and HSR-TDMA. As illustrated in Figure 11, the uplink and downlink multihop delays of GSR-TDMA are still shorter than those of the other two MAC protocols under UNs' random distribution and density, the same as Cases I, II, and III. Based on the RSR and the MHD performances, it can be concluded that GSR-TDMA can be also more advantageous than the other two MAC protocols for Case IV.

\section{Conclusions}

GSR-TDMA is designed for use in a centralized multihop UASN that takes advantage of spatiotemporal uncertainty. 

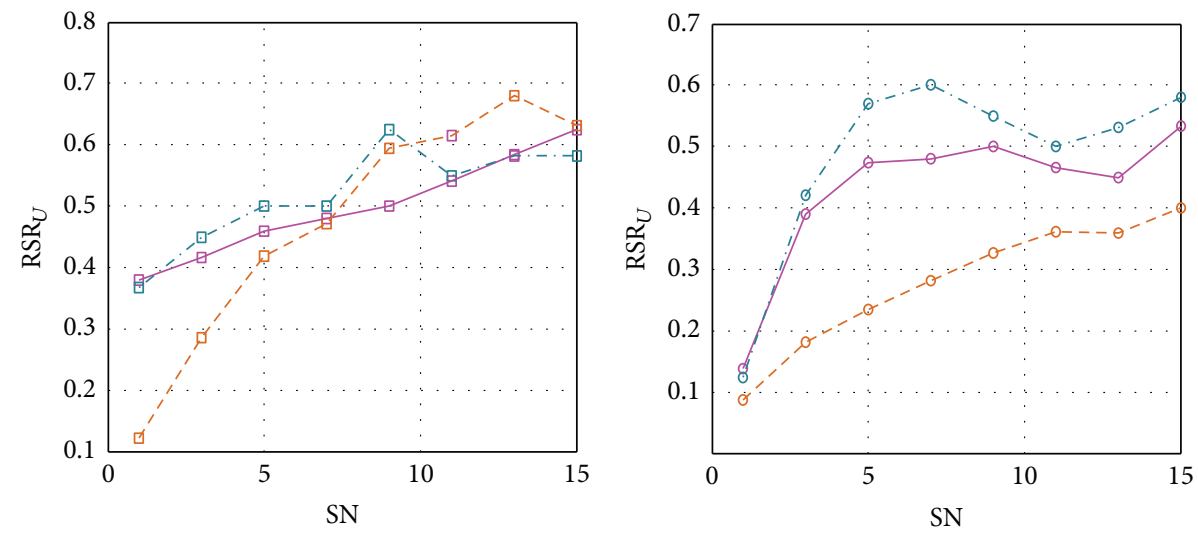

$$
\begin{aligned}
& \square \text { GSR-TDMA, } m=3 \\
& -\square-\text { HSR-TDMA, } m=3 \\
& \square-\text { CMAC, } m=3
\end{aligned}
$$

(a)

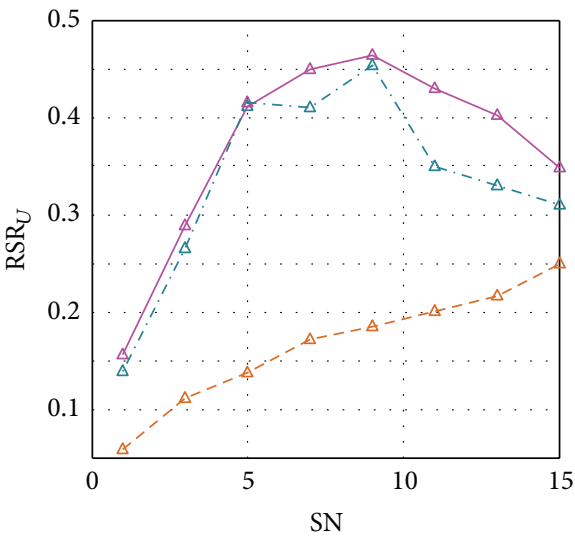

$\triangle$ GSR-TDMA, $m=7$

$-\triangle-$ HSR-TDMA, $m=7$

$\triangle-$ CMAC, $m=7$

(c)

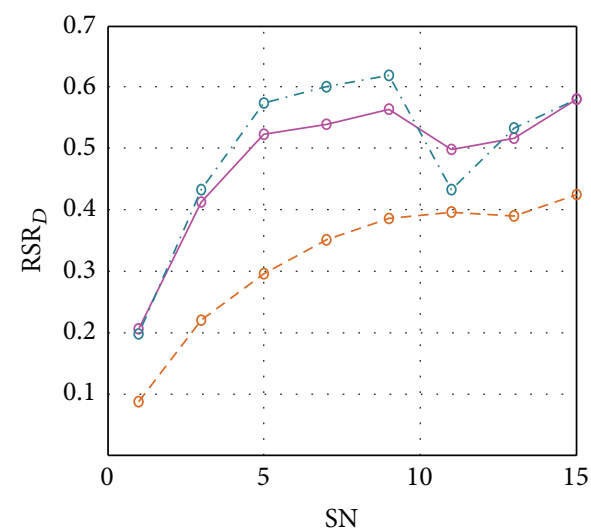

- GSR-TDMA, $m=5$

$-\ominus-$ HSR-TDMA, $m=5$

- - CMAC, $m=5$

(e)
- - GSR-TDMA, $m=5$

$-\theta-$ HSR-TDMA, $m=5$

- - CMAC, $m=5$

(b)

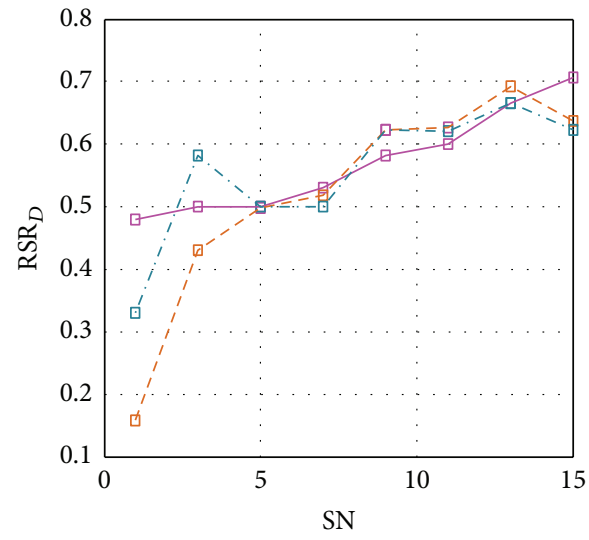

$\square-$ GSR-TDMA, $m=3$

- HSR-TDMA, $m=3$

$\checkmark-$ CMAC, $m=3$

(d)

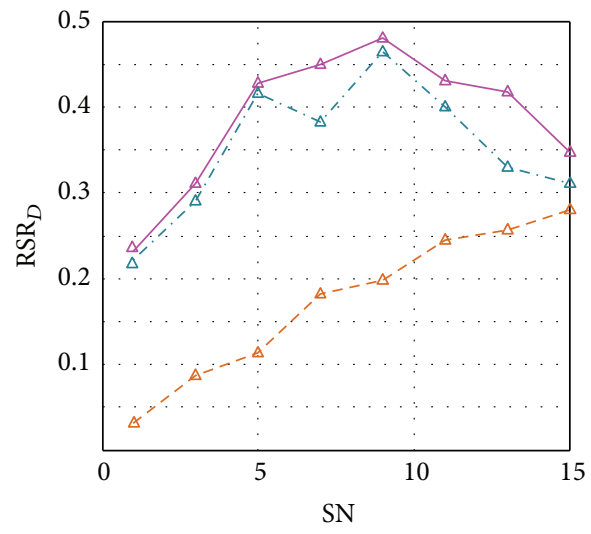

$\triangle$ GSR-TDMA, $m=7$
$-\triangle-$ HSR-TDMA, $m=7$
$\triangle-$ CMAC, $m=7$

(f)

FiguRe 10: The RSR of GSR-TDMA, HSR-TDMA, and C-MAC for Case IV. (a) $\operatorname{RSR}_{U}$ for $m=3$, (b) $\operatorname{RSR}_{U}$ for $m=5$, (c) $\operatorname{RSR}_{U}$ for $m=7$, (d) $\mathrm{RSR}_{D}$ for $m=3$, (e) $\operatorname{RSR}_{D}$ for $m=5$, and (f) $\operatorname{RSR}_{D}$ for $m=7$. 

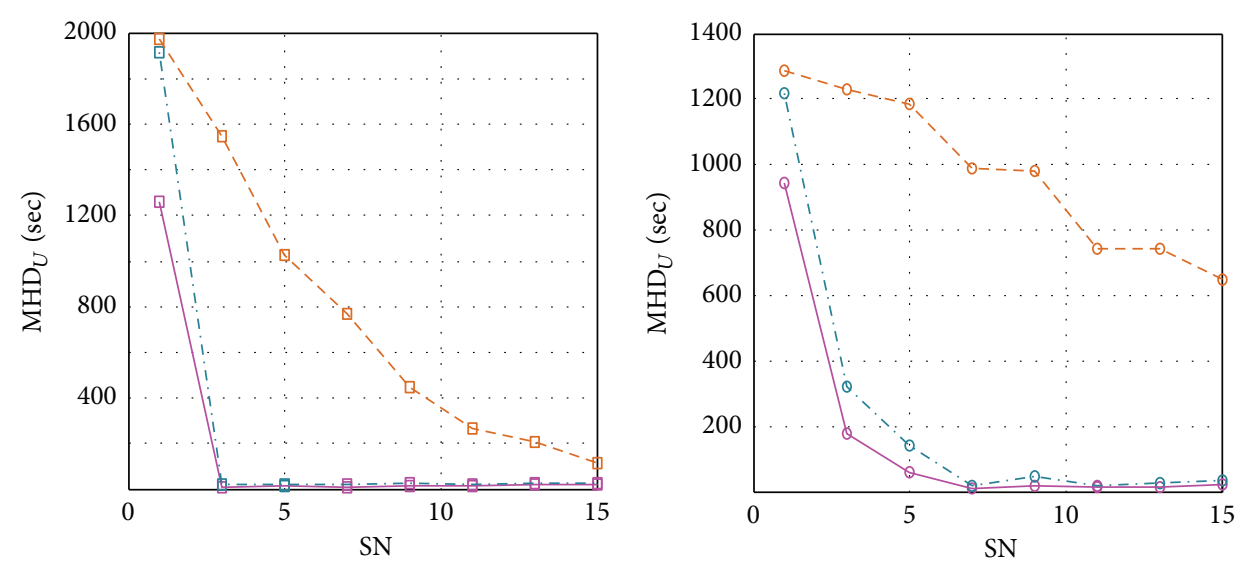

$$
\begin{aligned}
& \square \text { GSR-TDMA, } m=3 \\
& -\square-\text { HSR-TDMA, } m=3 \\
& \square-\text { CMAC, } m=3
\end{aligned}
$$

(a)

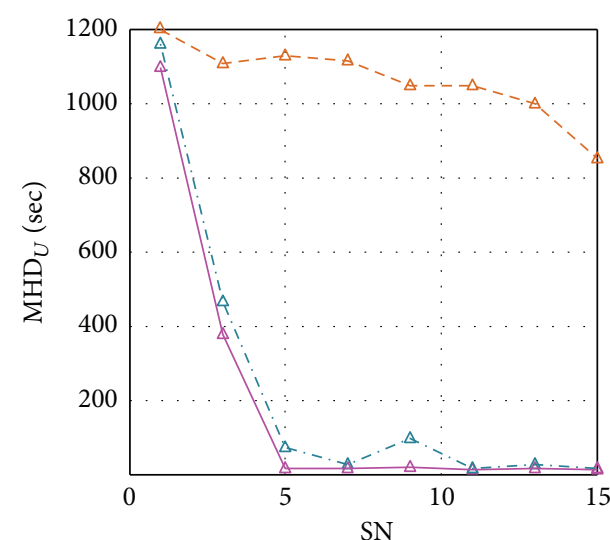

$$
\begin{aligned}
& \triangle-\text { GSR-TDMA, } m=7 \\
& -\Delta-\text { HSR-TDMA, } m=7 \\
& -\Delta-\text { CMAC, } m=7
\end{aligned}
$$

(c)

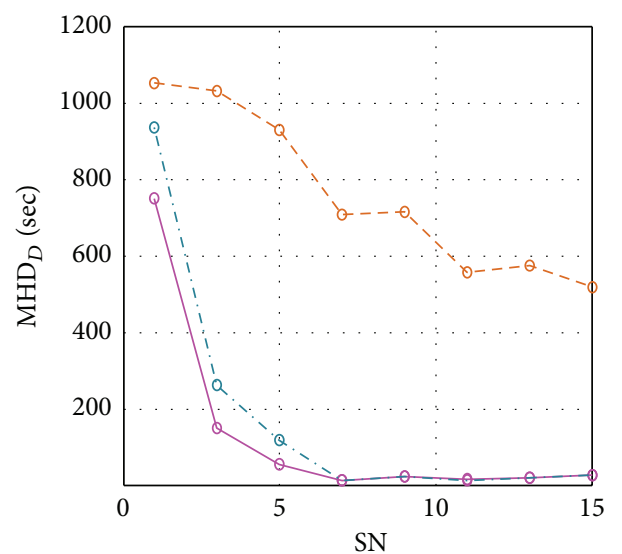

SN

$$
\begin{aligned}
& - \text { GSR-TDMA, } m=5 \\
& -\ominus-\text { HSR-TDMA, } m=5 \\
& -0 \text { CMAC, } m=5
\end{aligned}
$$

(e)
- - GSR-TDMA, $m=5$

$-\ominus-$ HSR-TDMA, $m=5$

- o- CMAC, $m=5$

(b)

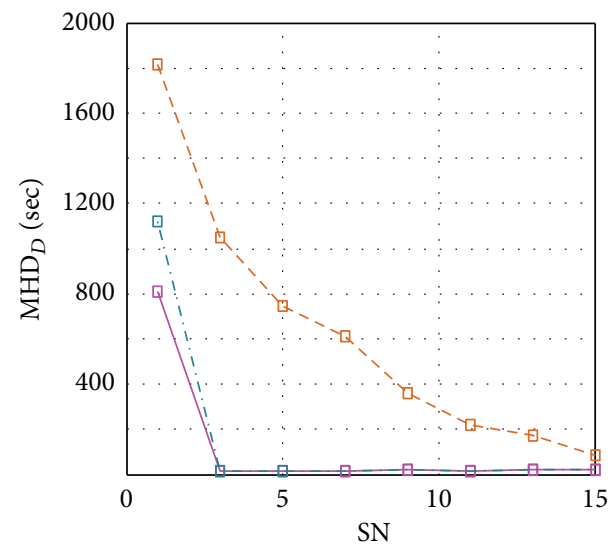

$$
\begin{aligned}
& -\square \text { GSR-TDMA, } m=3 \\
& -\boxminus-\text { HSR-TDMA, } m=3 \\
& -\square \text { CMAC, } m=3
\end{aligned}
$$

(d)

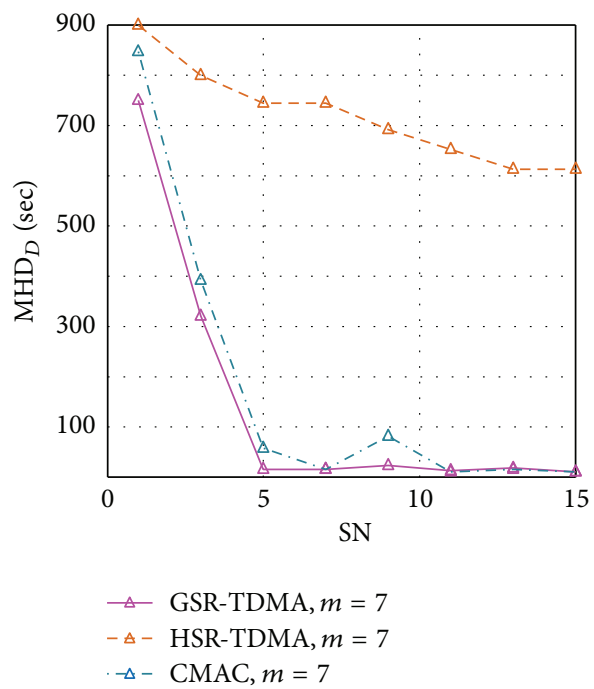

(f)

FIgure 11: The MHD of GSR-TDMA, HSR-TDMA, and C-MAC for Case IV. (a) $\mathrm{MHD}_{U}$ for $m=3$, (b) $\mathrm{MHD}_{U}$ for $m=5$, (c) MHD for $m=7$, (d) $\mathrm{MHD}_{D}$ for $m=3$, (e) $\mathrm{MHD}_{D}$ for $m=5$, and (f) $\mathrm{MHD}_{D}$ for $m=7$. 
For GSR-TDMA, collision-free transmission scheduling for all UNs is determined after setting a geometric map. The interhop and intrahop scheduling for GSR-TDMA can increase the number of UNs that send packets at the same time, and the extensive simulation in this work verifies these results. The simulation experiments are executed by varying the number of hops, the size of the packet train, and the period of packet generation. The results indicate that GSR-TDMA mostly outperforms C-MAC, irrespective of the conditions, except when the number of hops is less than five under UNs' random distribution and density. GSR-TDMA results in a 15\% higher RSR and 30\% lower MHD at maximum than C-MAC. In addition, GSR-TDMA outperforms HSRTDMA in a large percentage of the cases, and GSR-TDMA provides better performance than HSR-TDMA (i.e., a 50\% higher RSR and lower MHD by an order of $10^{2}$ than that at maximum). In the case of the error-occurring case, the performance of the three MAC protocols is remarkably degraded as the error rate increases, and the performance of the three MAC protocols still holds, regardless of the conditions. Thus, it can be concluded that GSR-TDMA that transmits packet trains can be more favorable as a suboptimal solution than the two previous approaches used for a multihop UASN.

\section{Parameter Definitions}

\begin{tabular}{|c|c|}
\hline$N$ : & The number of all UNs \\
\hline$m:$ & The number of hops \\
\hline$n:$ & An index of an interhop group $(0 \leq n \leq 2)$ \\
\hline$h:$ & An index of a hop $(0 \leq h \leq m)$ \\
\hline$N_{h}:$ & $\begin{array}{l}\text { The number of all UNs belonging to Hop } \\
h\left(\sum_{h=1}^{m} N_{h}=N\right)\end{array}$ \\
\hline$k$ : & An index of a subhop belonging to \\
\hline & belonging to Hop $h\left(1 \leq k \leq 2^{h}, h>1\right)$ \\
\hline$N_{h, k}:$ & The number of all UNs belonging to \\
\hline & Subhop $k$ in Hop $h\left(\sum_{k=1}^{2^{h}} N_{h, k}=N_{h}\right)$ \\
\hline$j:$ & $\begin{array}{l}\text { An index of a UN belonging to Subhop } \\
k\left(1 \leq j \leq N_{h, k}\right)\end{array}$ \\
\hline $\mathrm{PD}_{h, k, j}:$ & $\begin{array}{l}\text { The propagation delay between } \mathrm{UN} j \text { and } \\
\mathrm{UN} j \text { 's furthest neighboring UN within } \\
\text { the communication coverage of } j\end{array}$ \\
\hline $\mathrm{LEN}_{h}$ : & $\begin{array}{l}\text { The maximum number of packets of a UN } \\
\text { according to } h\end{array}$ \\
\hline TD: & $\begin{array}{l}\text { The transmission delay to transmit a } \\
\text { packet }\end{array}$ \\
\hline$T_{G}$ & The guard time \\
\hline & An index of a cycle. \\
\hline
\end{tabular}

\section{Conflict of Interests}

The authors declare that there is no conflict of interests regarding the publication of this paper.

\section{Acknowledgments}

This work was conducted as a part of the research project of "Development of the Wide-Area Underwater Mobile
Communication Systems" financially supported by the Ministry of Land, Transport and Maritime Affairs (MLTM) of Korea.

\section{References}

[1] I. F. Akyildiz, D. Pompili, and T. Melodia, "Underwater acoustic sensor networks: research challenges," Ad Hoc Networks, vol. 3, no. 3, pp. 257-279, 2005.

[2] Z. Jiang, "Underwater acoustic networks," International Journal of Intelligent Control and Systems, vol. 13, no. 3, pp. 152-161, 2008.

[3] M. Chitre, S. Shahabudeen, and M. Stojanovic, "Underwater acoustic communications and networking: recent advances and future challenges," Marine Technology Society Journal, vol. 42, no. 1, pp. 103-116, 2008.

[4] R. Otnes, A. Asterjadhi, P. Casari et al., Underwater Acoustic Networking Techniques, Springer, Heidelberg, Germany, 2012.

[5] R. J. Urick, Principle of Underwater Sound, McGraw-Hill, New York, NY, USA, 3rd edition, 1983.

[6] Z. Guan, T. Melodia, and D. Yuan, "Stochastic channel access for underwater acoustic networks with spatial and temporal interference uncertainty," in Proceedings of the 7th ACM International Conference on Underwater Networks and Systems (WUWNet '12), no. 18, pp. 1-8, ACM, Los Angeles, Calif, USA, November 2012.

[7] N. Chirdchoo, W.-S. Soh, and K. C. Chua, "Aloha-based MAC protocols with collision avoidance for underwater acoustic networks," in Proceedings of the 26th IEEE International Conference on Computer Communications (IEEE INFOCOM '07), pp. 22712275, IEEE, Anchorage, Alaska, USA, May 2007.

[8] M. Molinks and M. Stojanovic, "Slotted FAMA: a MAC protocol for underwater acoustic networks," in Proceedings of the IEEE OCEANS 2006 Asia Conference, pp. 1-7, Singapore, May 2007.

[9] S. Shahabudeen, M. Chitre, and M. Motahi, "A multi-channel MAC protocol for AUV networks," in Proceedings of the OCEANS-Europe, pp. 1-6, IEEE, Aberdeen, Scotland, June 2007.

[10] A. Syed, W. Ye, J. Heidemann, and B. Krishnamachari, "Understanding spatio-temporal uncertainty in medium access with ALOHA protocols," in Proceedings of the 2nd Workshop on Underwater Networks (WuWNet '07), pp. 41-48, ACM, Montreal, Canada, September 2007.

[11] H. T. Nguyen, S. Y. Shin, and S. H. Park, "State-of-the-art in MAC protocols for underwater acoustics sensor networks," in Proceedings of the EUC 2007 Workshops: TRUST, WSOC, NCUS, UUWSN, USN, ESO, and SECUBIQ, pp. 482-493, Taipei, Taiwan, December 2007.

[12] E. M. Sozer, M. Stojanovic, and J. G. Proakis, "Underwater acoustic networks," IEEE Journal of Oceanic Engineering, vol. 25, no. 1, pp. 72-83, 2000.

[13] D. Pompili, T. Melodia, and I. F. Akyildiz, "A CDMA-based medium access control for underwater acoustic sensor networks," IEEE Transactions on Wireless Communications, vol. 8, no. 4, pp. 1899-1909, 2009.

[14] R. Diamant, M. Pinkhasevich, and I. Achrak, "A novel spatially shared TDMA protocol and quality measure for ad hoc underwater acoustic network," in Proceedings of the International Conference on Advanced Information Networking and Applications Workshops (WAINA '09), pp. 1160-1165, IEEE, Bradford, UK, May 2009. 
[15] K. Kredo II, P. Djukic, and P. Mohapatra, "STUMP: exploiting position diversity in the staggered TDMA underwater MAC protocol," in Proceedings of the IEEE (INFOCOM '09), pp. 29612965, IEEE, Rio de Janeiro, Brazil, April 2009.

[16] Y. Guan, C.-C. Shen, and J. Yackoski, "MAC scheduling for high throughput underwater acoustic networks," in Proceedings of the IEEE Wireless Communications and Networking Conference (WCNC '11), pp. 197-202, Cancun, Mexico, March 2011.

[17] Y. Ma, Z. Guo, Y. Feng, M. Jiang, and G. Feng, "C-MAC: a TDMA-based MAC protocol for underwater acoustic sensor networks," in Proceedings of the International Conference on Networks Security, Wireless Communications and Trusted Computing (NSWCTC '09), vol. 1, pp. 728-731, IEEE, Wuhan, China, April 2009.

[18] R. Diamant and L. Lampe, "Spatial reuse time-division multiple access for broadcast ad hoc underwater acoustic communication networks," IEEE Journal of Oceanic Engineering, vol. 36, no. 2, pp. 172-185, 2011. 


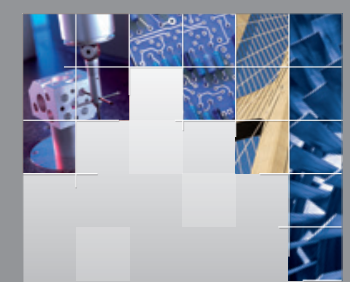

\section{Enfincering}
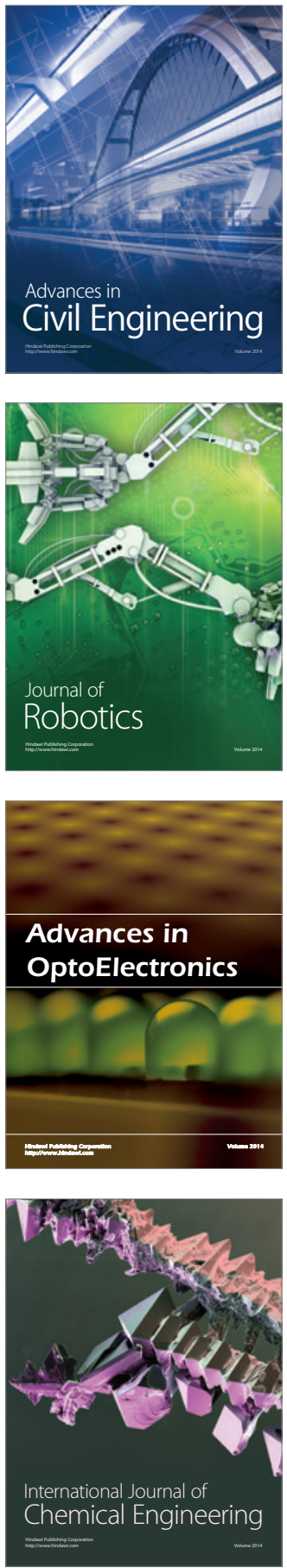

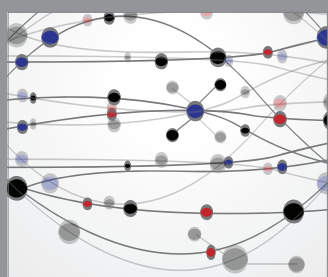

The Scientific World Journal

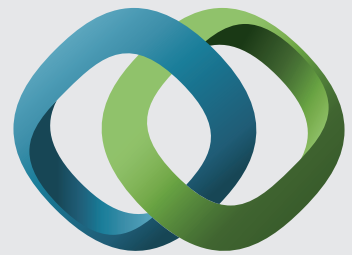

\section{Hindawi}

Submit your manuscripts at

http://www.hindawi.com
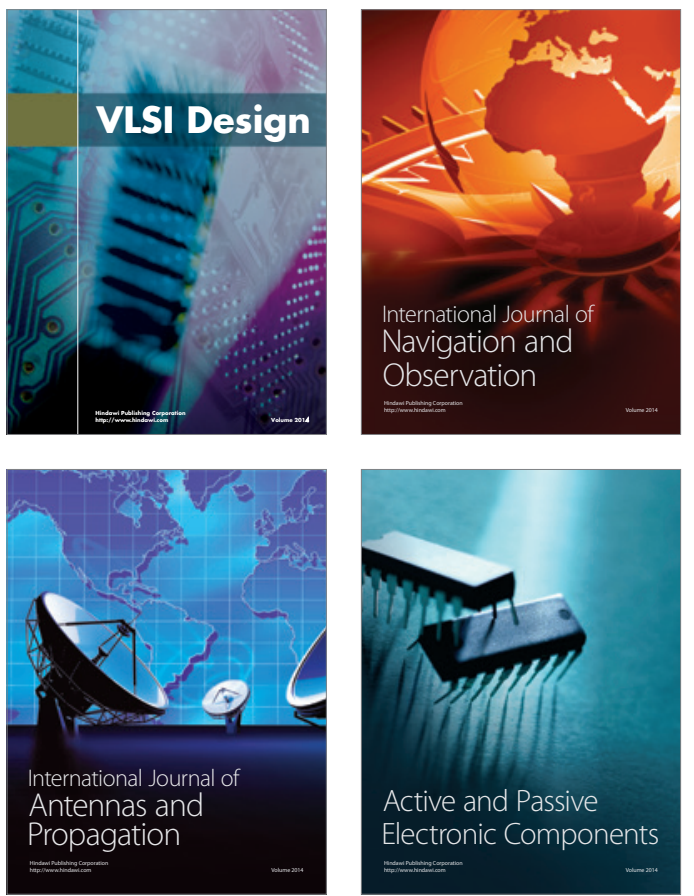
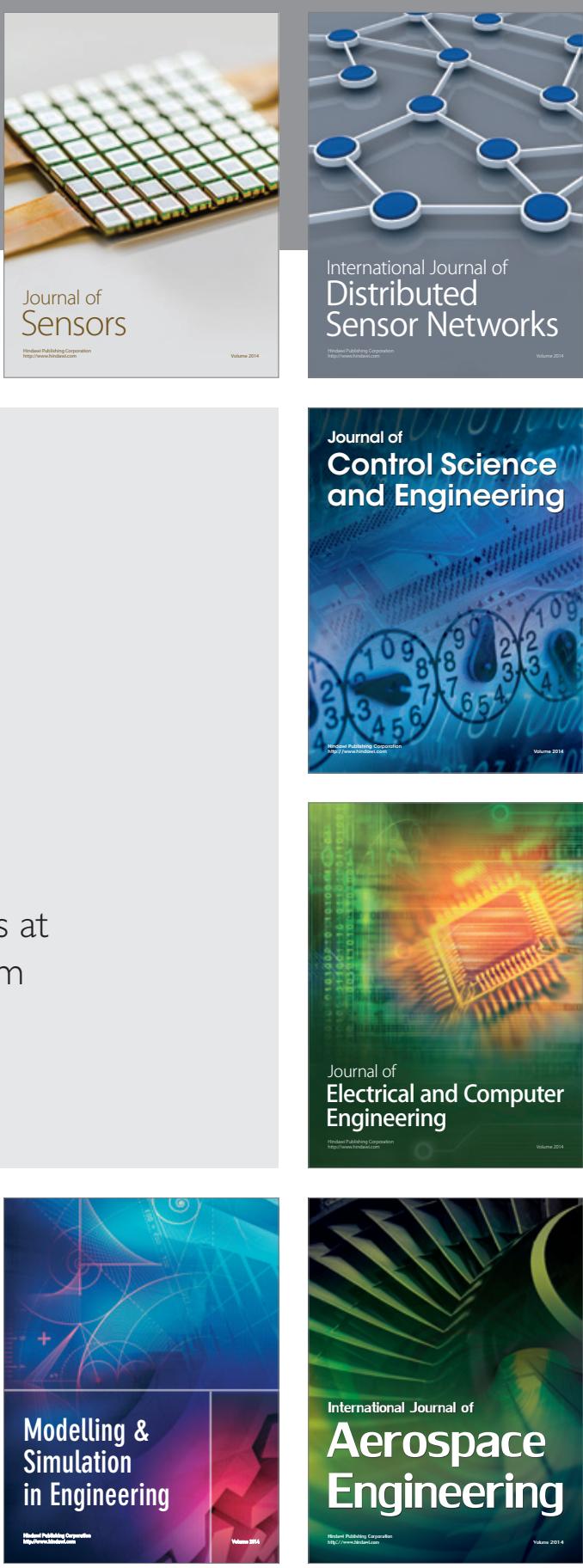

International Journal of

Distributed

Sensor Networks

Journal of

Control Science

and Engineering
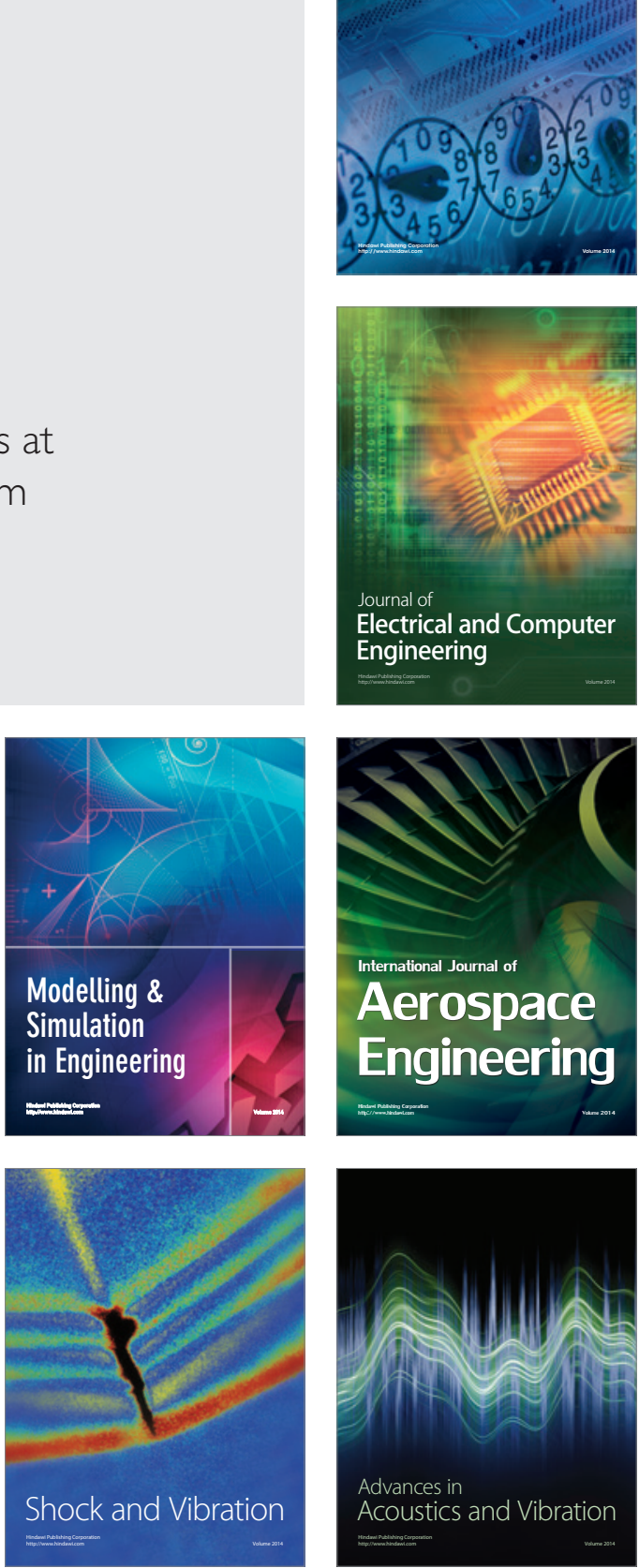